\title{
Comparison of channel-assisted minimally invasive repair and 3 common Achilles tendon restoration techniques
}

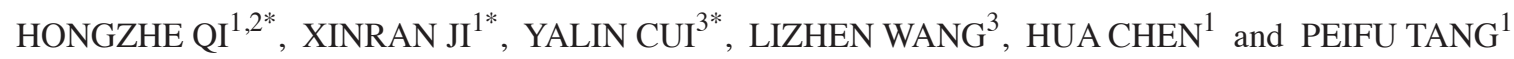 \\ ${ }^{1}$ Department of Orthopaedics, Chinese People's Liberation Army General Hospital, Beijing 100853; \\ ${ }^{2}$ Department of Orthopaedic Surgery, Chinese People's Liberation Army 306th Hospital, Beijing 100101; \\ ${ }^{3}$ Key Laboratory for Biomechanics and Mechanobiology, Ministry of Education, \\ School of Biological Science and Medical Engineering, Beihang University, Beijing 100191, P.R. China
}

Received October 16, 2017; Accepted March 23, 2018

DOI: $10.3892 /$ etm.2018.7075

\begin{abstract}
The present study aimed to investigate the biomechanical comparison of channel-assisted minimally invasive restoration and three common Achilles tendon restoration techniques in an in vitro model via a progressive rehabilitation program. The 42 porcine tendons were randomly assigned to the following six groups of tendons ( $\mathrm{n}=7 /$ group): Achillon, percutaneous Achilles repair system (PARS), Krackow, channel-assisted minimally invasive repair (CAMIR), CAMIR augmentation (CAMIR ${ }^{+}$), CAMIR-5 (repair with No. 5 Ethibond suture). There was no significant difference in elongation among groups following the first 10 loading cycles, which consisted of 20-100 $\mathrm{N}$ at $1 \mathrm{~Hz}$. The elongation of the CAMIR group $(7.51 \pm 1.77 \mathrm{~mm})$ was significantly longer than the Achillon group $(3.19 \pm 0.57 \mathrm{~mm})$ and PARS group $(3.73 \pm 0.66 \mathrm{~mm} ; \mathrm{P}<0.05)$ following 1,000 cycles. However, the CAMIR group exhibited no significant difference vs. the Krackow $(7.32 \pm 1.09 \mathrm{~mm})$ and CAMIR $^{+}$groups $(7.11 \pm 1.50 \mathrm{~mm})$ following 1,000 cycles. Following 2,000 cycles, there was no significant difference between the CAMIR-5 $(7.99 \pm 1.68 \mathrm{~mm})$ group, and the Achillon $(3.19 \pm 0.57 \mathrm{~mm})$ and PARS groups $(3.73 \pm 0.66 \mathrm{~mm})$. At the point of restoration construct failure, the total cycles of the CAMIR group (median, 1,000; range, 1,000-1,000) were significantly less than the Achillon group (median, 2,000; range, 2,000-2,013) and PARS group (median, 2,000; range, 2,000-2,010; $\mathrm{P}<0.05$ ), but had no significant difference compared with the Krackow group (median, 1,000; range, 1,000-1,000) and $\mathrm{CAMIR}^{+}$group
\end{abstract}

Correspondence to: Dr Hua Chen or Dr Peifu Tang, Department of Orthopaedics, Chinese People's Liberation Army General Hospital, 28 Fuxing Road, Wukesong, Beijing 100853, P.R. China

E-mail: chenhua0270@126.com

E-mail: tangpf301@163.com

*Contributed equally

Key words: Achilles tendon, percutaneous surgery, open surgery, biomechanical test, rupture
1,000 (median, 1,000; range, 1,000-1,004). There was also no significant difference between the CAMIR-5 group (median, 2,000; range, 2,000-2,000), and the Achillon group (median, 2,000; range, 2,000-2,013) and PARS group (median, 2,000; range, 2,000-2,010). Restricted by the strength of suture, the one-suture CAMIR restoration technique was weaker than the three-suture Achillon and PARS restoration techniques, but there was no significant difference with the open Krackow restoration technique, which provides a reliable mechanical strength for repairing. CAMIR has an advantage of reducing the risk of suture reactivity.

\section{Introduction}

The Achilles tendon is one of the thickest and strongest tendons in the human body, but is also one of the most frequently ruptured (1). Ganestam et al (2) recently demonstrated that the probability of acute Achilles tendon rupture has increased from 26.95 to 31.17 out of $10^{5}$ individuals between 1994 and 2013 in Denmark. However, there is currently no definitive consensus on whether surgery should be chosen to repair the ruptured acute Achilles tendon. Surgical treatment is often preferred in healthy and active patients (3). Khan et al (3) published a meta-analysis of randomized controlled blind prospective trials suggesting that conservative treatment with surgical treatment can reduce the incidence of Achilles tendon rupture. However, as surgical incision complications are more likely, incision complications can be reduced by percutaneous suturing.

The systematic analysis based on randomized controlled trials published by Wilkins and Bisson (4) came to the same conclusion. A meta-analysis based on a randomized controlled trial published by Deng et al (5) suggested that surgical treatment effectively reduced the re-rupture rate and may be a better choice for the treatment of acute Achilles tendon rupture. However, a meta-analysis based on randomized controlled trials published by Sororeanu et al (6) suggested that conservative treatment did not increase the rate of Achilles tendon rupture and, at the same time, avoided incision complications. Surgical treatment is often preferred in healthy and active patients (7). It is recommended that conservative treatment should be considered if patient require functional rehabilitation (6). Additionally, surgical repair should be preferred at 
centers that do not employ early range-of-motion protocols as it has been revealed to decrease the re-rupture risk in such patients (6).

For surgical treatment, open surgery, small incision or percutaneous suturing are also controversial (7). Del Buono et al (8) indicated that percutaneous techniques exhibit the following advantages: Low complication rate, reduced operating time, accelerated rehabilitation, reduced cost and improved aesthetic results. However, they may lead to a higher rate of recurrence than open surgical restoration techniques. However, Li et al (9) demonstrated that there was no statistically significant difference in recurrence rate between percutaneous treatment and open surgery. Sural nerve involvement was also a major difficulty in percutaneous minimally invasive techniques, leading to damage and inflexibility (10). Due to the small incision and limited exposure range during percutaneous minimally invasive surgery, the sural nerve cannot be directly viewed (8). The sural nerve at the proximal side of the lateral malleolus is associated with the lateral side of the Achilles tendon (11). Suture needles can easily damage the sural nerves when they are blindly suturing Achilles tendons (11).

Certain techniques have been used to avoid the risk of nerve injury for doctors, including endoscopy-assisted percutaneous restoration (8), internal splinting technique (11) and the modified Mayo needle technique (12-14). However, there was no method that reduced the risk of nerve injury and achieved a satisfactory clinical result for patients. A study demonstrated that the duration of surgery was increased with the use of endoscopic techniques (12). When using the modified Mayo needle technique, a lateral incision tends to allow visualization of the nerve (13). In the process of percutaneous minimally invasive suture, because the sural nerve travels between the Achilles tendon and the external malleolus, the nerve is not exposed and protected during the surgery, and the nerve may be damaged through the nerve during the blind suture (14). The surgical incision is relatively increased and the operation time is expended accordingly. Therefore, the surgery is cumbersome and the problem of sural nerve injury cannot be solved simply and quickly. A channel-assisted minimally invasive repair (CAMIR) (15) that was recently devised by the present authors, has achieved satisfactory clinical effects. The present study aimed to biomechanically compare two commercially available, minimally invasive percutaneous techniques and a gold standard open Achilles repair $(10,16)$ with the techniques of CAMIR and CAMIR augmentation during a simulated, progressive rehabilitation program. Therefore, the present study aimed to investigate the biomechanical comparison of channel-assisted minimally invasive restoration and three common Achilles tendon restoration techniques in an in vitro model via a progressive rehabilitation program.

\section{Materials and methods}

Animals. A total of 21 12-month-old skeletally mature male pigs (weight, $50 \pm 10 \mathrm{~kg}$; Animal Laboratory, General Hospital of People's Liberation Army, Beijing, China) were housed in cages and exposed to a 12-h light/dark cycle. Pigs were fed regularly with commercially available pig food. The right and left hind legs were used for the present study. Pigs were housed between 23 and $25^{\circ} \mathrm{C}, 65-75 \%$ humidity. The Ethics Committee for Experiments on Animals of General Hospital of People's Liberation Army approved all procedures performed in the present study.

Experimental design. A total of 42 fresh porcine Achilles tendons were obtained from 21 pigs following their sacrifice, stored at $-20^{\circ} \mathrm{C}$ and thawed for $12 \mathrm{~h}$ by infiltrating $0.9 \% \mathrm{NaCl}$ prior to testing at room temperature. The proximal tenotomy, simulated midsubstance rupture, was made at $4 \mathrm{~cm}$ over the end of the plantar flexor tendon. The distal tenotomy was made $\sim 2 \mathrm{~cm}$ below the end of the plantar flexor tendon. Moisture of the tendon was maintained with saline sprays during the preparation and testing. The width and thickness of all specimens were measured at the proximal end and the suture end using vernier calipers (Guilin Guanglu Digital Measurement \& Control Co., Ltd., Guilin, China). The 42 tendons were randomly assigned into six groups $(n=7$ each) and were subjected to different Achilles restoration techniques: Krackow (16), Achillon (17,18), percutaneous Achilles repair system (PARS), channel-assisted minimally invasive repair (CAMIR) (15), CAMIR augmentation $\left(\mathrm{CAMIR}^{+}\right)$and CAMIR-5 (repair with No. 5 Ethibond suture).

Simulated tears were subsequently repaired using the randomly assigned restoration technique (Fig. 1). This figure presents all Achilles restoration techniques, however as the only difference between CAMIR and CAMIR-5 is the size of the suture, one image is used to represent the two techniques. All surgical restorations were performed by a single foot and ankle fellowship-trained orthopedic surgeon. Suturing commenced $1 \mathrm{~cm}$ from the proximal edge of tendon specimens, and a constant $1 \mathrm{~cm}$ was maintained from the proximal to distal strand. The Achillon, PARS, Krackow and CAMIR methods were applied using No. 2 Ethibond (Ethicon, Inc., Cincinnati, OH, USA). CAMIR ${ }^{+}$was applied using No. 2 Ethibond (Ethicon, Inc.) sutures augmented with intermittent sutures of absorbable Vicryl 2-0. CAMIR-5 was applied using No. 5 Ethibond (Ethicon, Inc.).

Biomechanics testing. A universal material testing dynamometer (Electro Puls E10000; Instron, Norwood, MA, USA) was used to cyclically load the tendons. Following the completion of suture-graft constructs, each specimen was mounted below the dynamometer with the non-sutured end of the tendon wrapped with gauze to eliminate slippage while secured in a custom clamp. The free strands of the suture were tied with ' 8 ' square knots over a custom bar of the universal material testing machine, mimicking a suture post in vivo (19-21). The non-sutured end of the tendon was in close proximity to the bar to minimize elongation of the suture. The whole suture constructs, from below the bar to above the clamp, were maintained at a standard length of $4 \mathrm{~cm}$ for testing (Fig. 2). Each tendon was pre-tensioned to $50 \mathrm{~N}$ for $2 \mathrm{~min}$ to remove slack from the construct. Each specimen was subjected to a loading protocol representative of a progressive, postoperative rehabilitation program (3,000 total cycles) consisting of three cyclic loading stages of 1,000 cycles at $1 \mathrm{~Hz}$ as follows: i) 20-100 N, ii) $20-191 \mathrm{~N}$ and iii) 20-369 N. Sutures that were maintained following all 1,000 cycles of loading were subsequently pulled to failure at a rate of $25 \mathrm{~mm} / \mathrm{sec}$ for each cyclic loading stage. Loads were selected to mimic a progressive, 


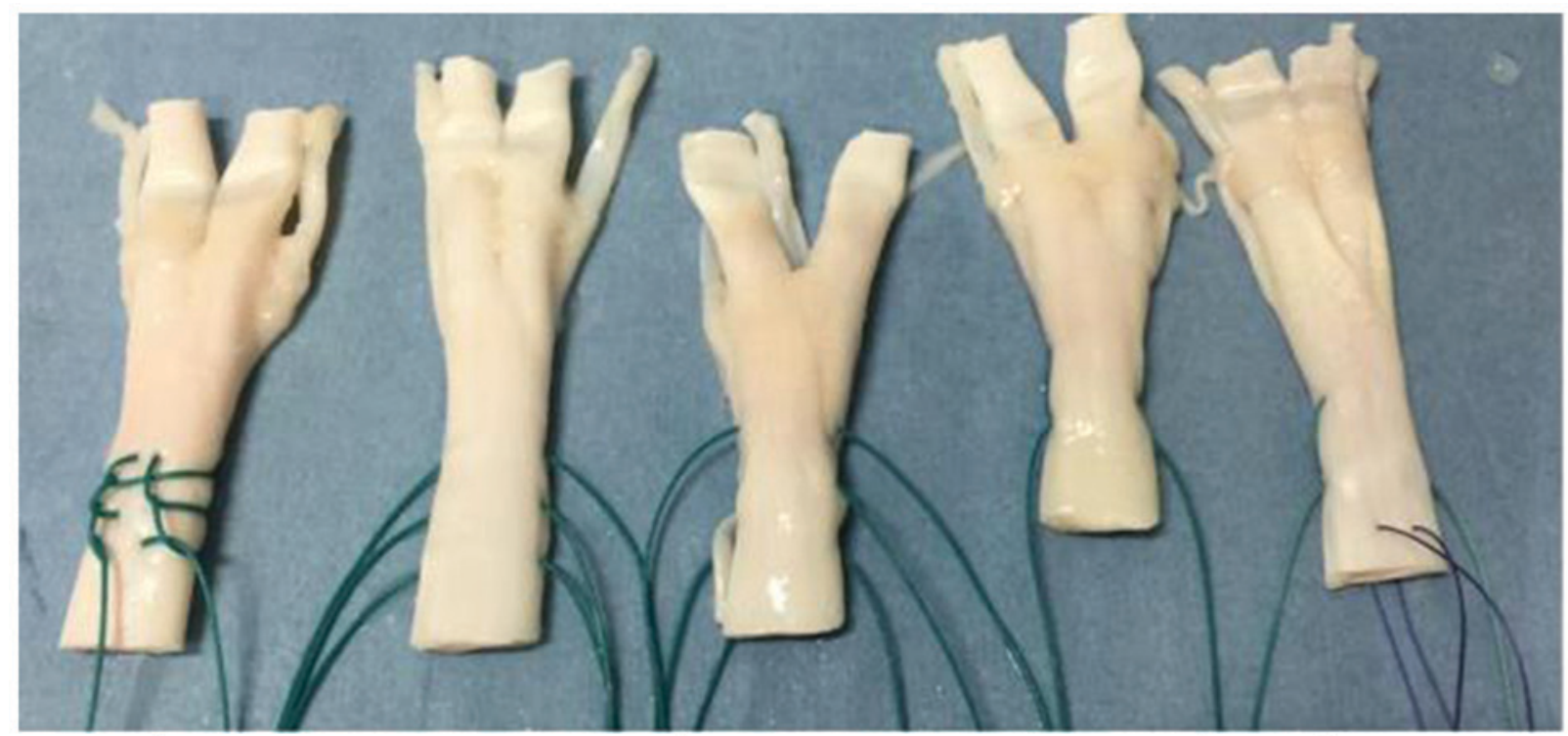

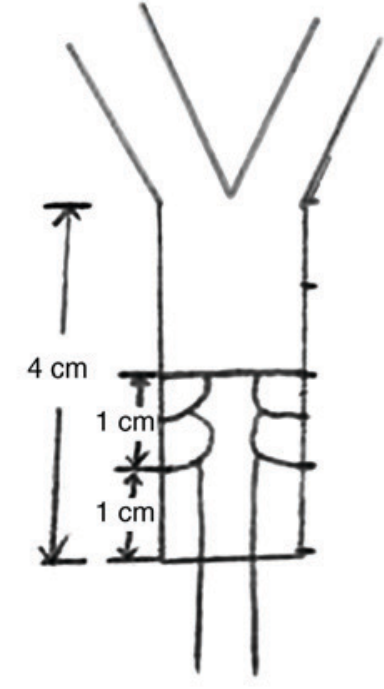

A

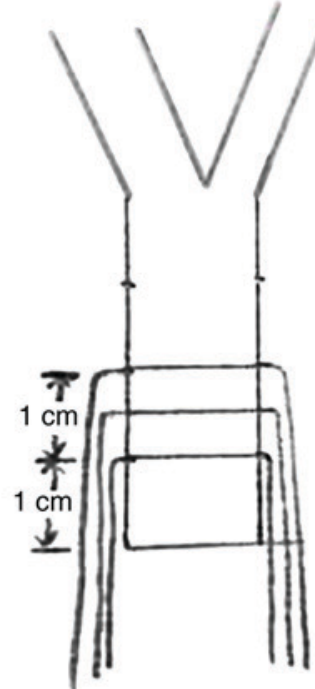

B

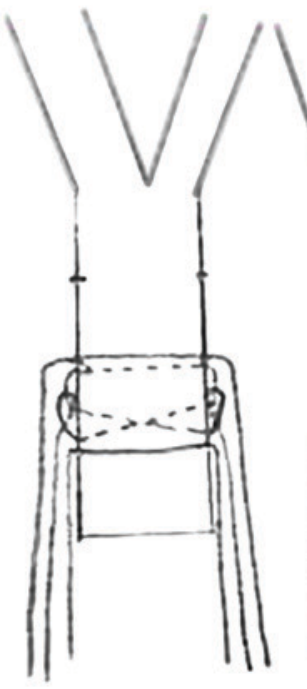

C

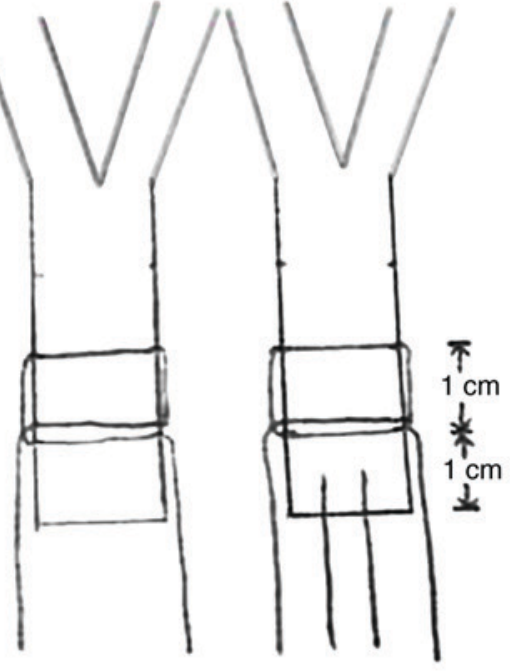

E

Figure 1. Photographs and simplified schematic diagrams illustrating the five different repair constructs and suture configurations. (A) Krackow repair, (B) Achillon repair, (C) percutaneous Achilles repair system repair, (D) CAMIR repair and (E) CAMIR augmentation repair. As the only difference between CAMIR and CAMIR-5 is the size of the suture, one image is used to represent the two techniques. CAMIR, channel-assisted minimally invasive repair.

postoperative rehabilitation protocol and were based on previous literature describing load ranges experienced by the Achilles tendon during passive ankle flexion (20-100 N) and walking in a CAM walker with $(191 \mathrm{~N})$ and without $(369 \mathrm{~N})$ a 1-inch heel lift $(22,23)$. Failure was defined as tendon breakage, suture breakage or suture pullout. Cyclics and elongation were monitored and recorded continuously throughout testing by dynamometer. The mode of failure for each construct and the number of stitch knots of different techniques were also recorded.

Statistical analysis. The maximum number of cycles is expressed as the median (minimum, maximum). The analysis of variance was used for the amount of stretch, and the expression was mean \pm standard deviation. To detect the effect size of one sample for differences in repaired elongation at the end of the first loading cycle, 7 specimens per group were required to achieve an $80 \%$ positive rate. The data of this calculation was based on the result of a pilot investigation and statistical analysis performed prior to completion of testing. Statistical analysis was performed using SPSS version 13.0 (SPSS, Inc., Chicago, IL, USA). One-way analysis of variance was applied to evaluate differences in the mean width and thickness of the tendon in each group, and the elongation following 10,1,000, 2,000 and 3,000 cycles. The Kruskal-Wallis test was used to assess if there was an association between the restoration technique and the number of cycles to total restorations failure. If a significant effect was noted, a post hoc Mann-Whitney U test (with Bonferroni corrections for multiple comparisons) was performed to assess differences between the different techniques. $\mathrm{P}<0.05$ was considered to indicate a statistically significant difference.

\section{Results}

Biomechanics test of Achilles tendon. There were no significant differences in Achilles tendon consistency among 


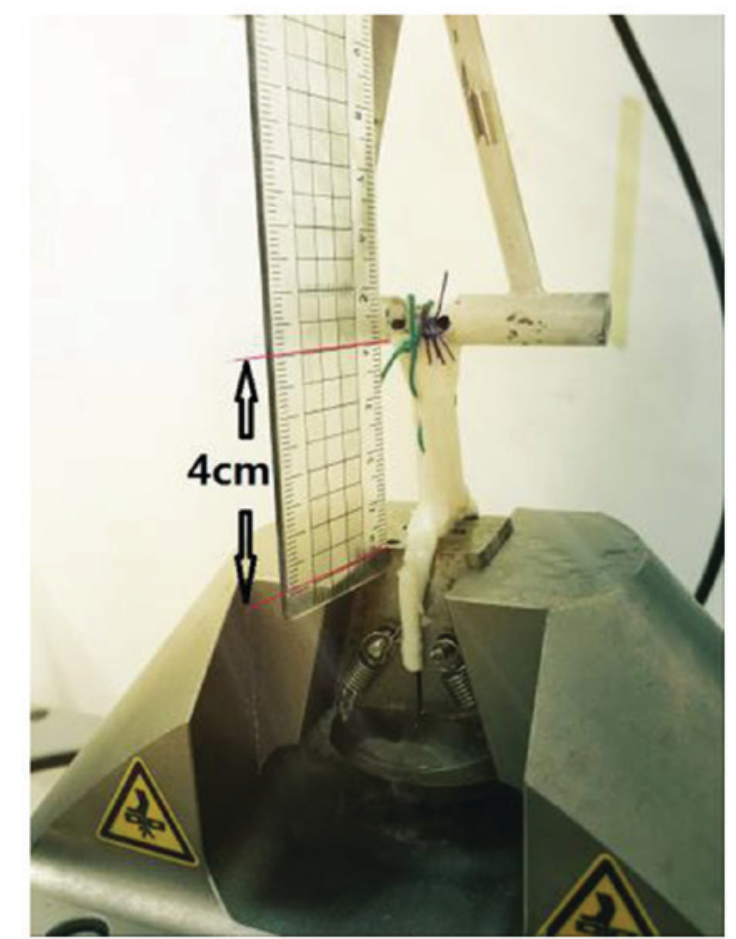

Figure 2. The whole suture constructs, from below the bar to above the clamp, were maintained at a standard length of $4 \mathrm{~cm}$ for testing.

groups (Table I). All restorations survived during the first stage of cyclic loading (20-100 N, 1,000 cycles), however 21 samples failed the second phase and 21 samples failed the third phase (3,000 cycles in all three stages; data not shown).

Failure time of cycle test. There was no significant difference in the number of cycles before restoration failure among the following restoration methods: Krackow, median 1,000 (range, 1,000-1,000); CAMIR, median 1,000 (range, 1,000-1,000); and $\mathrm{CAMIR}^{+}$median 1,000 (range, 1,000-1,004). However, they were significantly less than that observed in the 2 percutaneous repair methods: Achillon, median 2,000 (range, 2,000-2,013); and PARS median 2,000 (range, 2,000-2,010; $\mathrm{P}<0.05$; Fig. $3 \mathrm{~A}$ ). In addition, there was no significant differences in cycles between CAMIR-5 (median, 2,000; range, 2,000-2,003), and Achillon and PARS (Fig. 3B).

Tendons in the CAMIR group are repaired with the No. 2 Ethibond suture test. Following the 10th cycle of cyclic loading, CAMIR and CAMIR ${ }^{+}$restoration exhibited a mean elongation of $2.16 \pm 1.12$ and $2.03 \pm 0.93 \mathrm{~mm}$ respectively, which were not significantly different than Krackow $(1.97 \pm 0.56 \mathrm{~mm})$, Achillon $(1.67 \pm 0.74 \mathrm{~mm})$ or PARS $(2.02 \pm 1.03 \mathrm{~mm})$. Following the 1,000 th cycle, the mean elongation of CAMIR $(7.51 \pm 1.77 \mathrm{~mm})$ and $\mathrm{CAMIR}^{+}(7.11 \pm 1.50 \mathrm{~mm})$ exhibited no significant difference compared with Krackow $(7.32 \pm 1.09 \mathrm{~mm})$, but were significantly longer than Achillon $(3.19 \pm 0.57 \mathrm{~mm})$ and PARS (3.73 $\pm 0.66 \mathrm{~mm} ; \mathrm{P}<0.05$; Table II).

Tendons in the CAMIR-5 group are repaired with the No. 5 Ethibond suture test. Following the 10th and 2,000th cycle of cyclic loading, the elongation of CAMIR-5 exhibited no significant difference vs. Achillon or PARS. However, at the 1,000th
Table I. The consistency of Achilles tendon.

\begin{tabular}{lcc}
\hline Group & Thickness $(\mathrm{mm})$ & Width $(\mathrm{mm})$ \\
\hline Achillon & $13.66 \pm 0.72$ & $4.63 \pm 0.58$ \\
PARS & $13.42 \pm 0.66$ & $4.37 \pm 0.54$ \\
Krackow & $13.56 \pm 0.68$ & $4.48 \pm 0.62$ \\
CAMIR $^{+}$ & $13.46 \pm 0.70$ & $4.51 \pm 0.56$ \\
CAMIR $^{+}$ & $13.61 \pm 0.72$ & $4.59 \pm 0.69$ \\
CAMIR-5 $^{-}$ & $13.46 \pm 0.68$ & $4.52 \pm 0.49$
\end{tabular}

Data are presented as the mean \pm standard deviation. PARS, percutaneous Achilles repair system repair; CAMIR, channel-assisted minimally invasive repair; CAMIR ${ }^{+}$, augmented CAMIR; CAMIR-5, CAMIR with No. 5 Ethibond suture.

Table II. Elongation following 10 and 1,000 cycles for the different repair techniques using No. 2 Ethibond suture.

\begin{tabular}{lcc}
\hline Group & $\begin{array}{c}\text { Elongation following } \\
10 \text { cycles }(\mathrm{mm})\end{array}$ & $\begin{array}{c}\text { Elongation following } \\
1,000 \text { cycles }(\mathrm{mm})\end{array}$ \\
\hline Achillon & $1.67 \pm 0.74$ & $3.19 \pm 0.57$ \\
PARS & $2.02 \pm 1.03$ & $3.73 \pm 0.66$ \\
Krackow & $1.97 \pm 0.56$ & $7.32 \pm 1.09^{\mathrm{a}}$ \\
CAMIR $^{2}$ & $2.16 \pm 1.12$ & $7.51 \pm 1.77^{\mathrm{a}}$ \\
CAMIR $^{+}$ & $2.03 \pm 0.93$ & $7.11 \pm 1.50^{\mathrm{a}}$ \\
\hline
\end{tabular}

Data are presented as the mean \pm standard deviation. ${ }^{a} \mathrm{P}<0.05$ vs. PARS. PARS, percutaneous Achilles repair system repair; CAMIR, channel-assisted minimally invasive repair; $\mathrm{CAMIR}^{+}$, augmented CAMIR.

cycle, the elongation of CAMIR-5 remained greater than that of Achillon and PARS restorations ( $\mathrm{P}<0.05$; Table III).

The number of sutures and knots. The primary mechanism of failure was suture break at the suture-tendon interface (Achillon, 3/7; PARS, 0/7; Krackow, 0/7; CAMIR, 1/7; CAMIR ${ }^{+}$, 0/7; CAMIR-5, 0/7) and breakage of knots (Achillon, 4/7; PARS, 7/7; Krackow, 7/7; CAMIR, 6/7; CAMIR+, 7/7; CAMIR-5, 7/7; Fig. 4). The number of stitch knots in each group was 3 (Achillon), 3 (PARS), 1 (Krackow), 1 (CAMIR) and 3 (CAMIR+; Fig. 5).

\section{Discussion}

Compared with open restoration, percutaneous techniques have many advantages, including minimized incisions, reduced surgery duration, improved cosmetic appearance and reduced rate of complication $(24,25)$. Compared with conservative treatment, it may also decrease the risk of rupture reoccurrence $(26,27)$. In the present study, CAMIR techniques were used easily through a minimal incision, on the tendon ends. The channel of CAMIR, which was designed by the authors of the present study, may reduce the risk of nerve injury, and achieved a satisfactory clinical effect (15). 

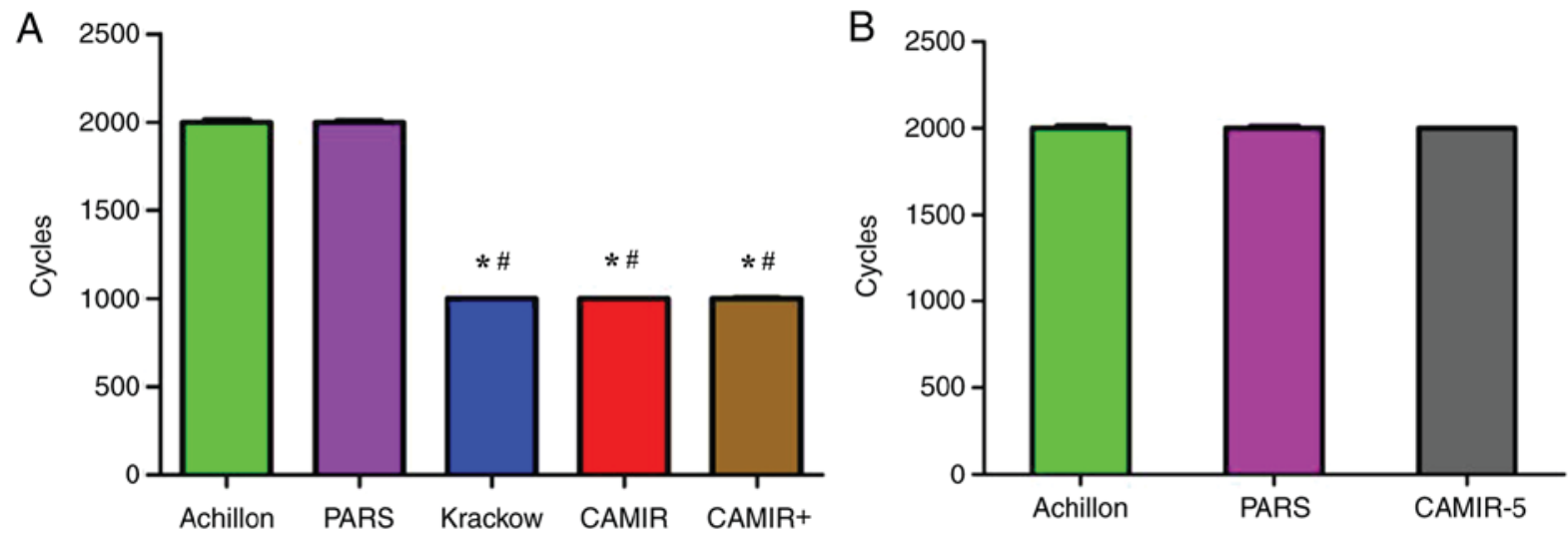

Figure 3. Number of cycles to total failure for the different repair techniques. (A) The groups repaired with No. 2 Ethibond suture. (B) CAMIR group repaired with No. 5 Ethibond suture compared with Achillon and PARS groups repaired with No. 2 Ethibond suture. Data are presented as the median and range. ${ }^{*} \mathrm{P}<0.05$ vs. Achillon; ${ }^{\text {P }}<0.05$ vs. PARS. CAMIR, channel-assisted minimally invasive repair; PARS, percutaneous Achilles repair system repair.

Early rehabilitation therapy is helpful to reduce postoperative complications of Achilles tendons, and yield a better effect of treatment for patients (28-30). However, following minimally invasive surgical treatment, surgeons often recommend cast immobilization to decrease the elongation of suture construct and the risk of failure (31). In order to provide reliable evidence for postoperative rehabilitation via the CAMIR technique, the present study simulated a progressive rehabilitation program with three stages of cyclic loading to compare the strength with the commonly used Krackow technique $(32,33)$ and the minimally invasive Achillon and PARS techniques, and evaluated the subsequent biomechanics $(25,29)$.

Unreliable stitching or incorrect rehabilitation treatment may make the suture constructs elongate easily, result in suture failure or making the Achilles tendon itself, elongate, which will lead to heel raise difficultly and claudication (28). These cause great physical and psychological harm to patients. Therefore, the elongation of the suture constructs under strain is a valuable test of biomechanics. The restoration of elongation was evaluated in the present study, instead of any direct measurement of repair site gapping as described in previous literature $(10,34)$. The elongation of Achilles tendons were recorded directly using an Instron biomechanical testing machine, including elongation of the entire tested construct, which may be in accordance with real-life observations in clinical practice. These measurements included the elongation of Achilles tendon tissue itself, the knot tightening or slipping, the suture being pulled through tendon and stretching of the suture material, which is similar to elongation in clinical practice. In the current study, eight square knots were used for each sample, and the knot size was the same, so that the baseline of the knot tightening or slipping was the same to offset the interference of this factor.

In addition, by suturing $1 \mathrm{~cm}$ from the proximal edge of tendon specimens, maintaining a $1 \mathrm{~cm}$ distance from the proximal to distal strand, and ensuring the non-sutured end of the tendon was in close proximity to the bar to minimize the elongation of suture, the baseline length of the sutures in each group was demonstrated to be basically the same, thus minimizing the influence of the stretching material factor. At the same time, to ensure that no significant differences in thicknesses and widths were identified between samples from all groups, each sample was $6 \mathrm{~cm}$ and the distance between the clamp and the bar was $4 \mathrm{~cm}$, thereby ensuring that the baseline of each sample was consistent. Therefore, the elongation measured during the biomechanical testing reflected the elongation of Achilles tendon tissue itself, as well as the sutures being pulled through tendon and stretching of the suture material (27). The total cycles following repair failure were another valuable index that reflected the strength of stitching technique. These reported values were recorded automatically by an Instron machine when the restoration failure occurred.

The comparisons of the biomechanical parameters in open and percutaneous restoration, and between the different percutaneous techniques were also conflicting. Few studies have assessed the simulated, progressive rehabilitation protocol. The majority of the studies consist of static experiments, which assess the maximum tensile strength or are failure experiments. Cyclic loading is less clinically assessed compared with the physiological state of the sputum. Mark-Christensen et al (28) compared the strength of tendons repaired with percutaneous techniques Achillon and PARS. Results demonstrated that the number of cycles prior to restoration failure in the Achillon group was significantly less than in the PARS groups, as the width and length of the Achilles tendons were detected by a differential variable reluctance transducer; the tendons were $2 \mathrm{~mm}$ in width and $9.5 \mathrm{~mm}$ in length. Otherwise, biomechanics testing demonstrated that the PARS group restorations resisted maximum load failure better than the Achillon group restorations (data not shown). Therefore, it was indicated that percutaneous repair techniques applying locking sutures offered a better strength of construction under cyclic and ultimate loads, compared with a restoration technique that used non-locking sutures. PARS was stronger than Achillon.

A previous study compared three commercially available, minimally invasive percutaneous techniques, Achillon, PARS and Speed Bridge, with an open Achilles restoration, modified Kessler, during a simulated and progressive rehabilitation program (31). Results demonstrated that the open restoration technique significantly reduced when compared with all minimally invasive percutaneous restoration methods following 250 cycles. There were no significant differences in mean displacements observed following 250 cycles between the Achillon, PARS, and Speed Bridge restorations, and 
Table III. Elongation following 10, 1,000 and 2,000 cycles for the CAMIR group repaired with No. 5 Ethibond suture, and the Achillon and PARS groups repaired with No. 2 Ethibond.

\begin{tabular}{lccc}
\hline Group & $\begin{array}{c}\text { Elongation following } \\
10 \text { cycles }(\mathrm{mm})\end{array}$ & $\begin{array}{c}\text { Elongation following } \\
1,000 \text { cycles }(\mathrm{mm})\end{array}$ & $\begin{array}{c}\text { Elongation following } \\
2,000 \text { cycles }(\mathrm{mm})\end{array}$ \\
\hline Achillon & $1.67 \pm 0.74$ & $3.19 \pm 0.57$ & $7.96 \pm 1.25$ \\
PARS & $2.02 \pm 1.03$ & $3.73 \pm 0.66$ & $8.15 \pm 1.32$ \\
CAMIR-5 & $1.59 \pm 0.56$ & $4.97 \pm 1.47^{\mathrm{a}}$ & $7.99 \pm 1.68$ \\
P-value & 0.561 & 0.01 & 0.971 \\
\hline
\end{tabular}

Data are presented as the mean \pm standard deviation. ${ }^{a} \mathrm{P}<0.05$ vs. PARS. PARS, percutaneous Achilles repair system repair; CAMIR-5, channel-assisted minimally invasive repair with No. 5 Ethibond suture.

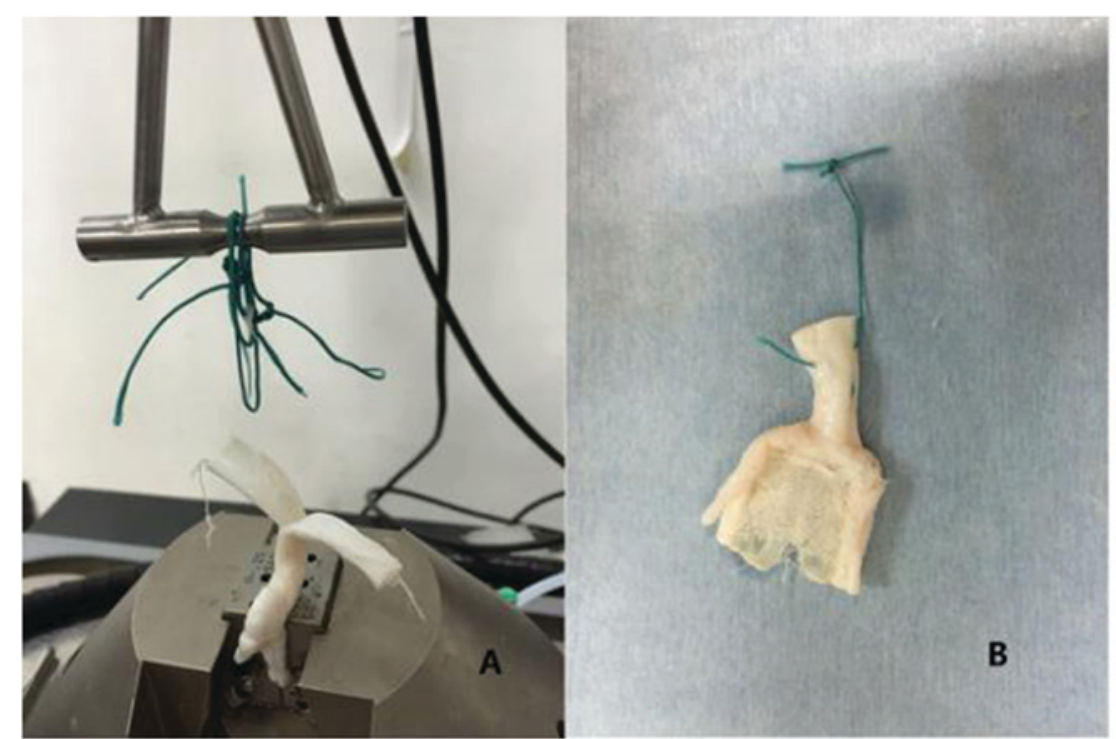

Figure 4. Primary mechanisms of failure. (A) Suture cutout at the suture-tendon interface. (B) Breakage of the knots.

there were no significant differences in the total number of cycles to failure between minimally invasive percutaneous restorations and open restorations. Clanton et al (31) revealed that minimally invasive percutaneous restoration techniques were susceptible to significant early restoration elongation when compared with open restorations, but the ultimate strengths of restorations (as indicated by failure cycles) were comparable among the open and percutaneous restoration techniques.

Lee et al (10) previously compared percutaneous Achillon, 4-strand Krackow, and an epitendinous augmented 4-strand Krackow restoration techniques during a simulated and progressive rehabilitation program, and it was reported that gap resistance was significantly better in augmented Krackow restorations (2,213 cycles to total failure gapping) vs. non-augmented restorations (1,268 cycles) and percutaneous restorations (102 cycles). Lee et al (10) also indicated that open restoration techniques (Krackow) making use of locking sutures offered a better construct of strength under cyclic loads compared with percutaneous restoration techniques (Achillon) that used only non-locking sutures. Otherwise, the cross-stitch weave augmentation significantly increased restoration strength and gap resistance. The current biomechanical experimental literature reported large differences in results and the conclusions were not consistent.

The present study indicated that there was no significant difference in the elongation at the end of the tenth cycle in all samples of Achilles tendons. And the majority of elongations did not occur in the initial 10 cycles, which contradicts the results of a previous study (27). The authors of the present study considered that the preloading of $50 \mathrm{~N}, 2$ min prior to the cyclic program Therefore, preloading may partially reduce the restoration elongation and deformation of tendons that occur during initial loading (first 10 cycles). All tendons were survived in the first stage of cyclic loading (20-100 N).

The Krackow, CAMIR and CAMIR ${ }^{+}$groups stitched by No. 2 sutures all failed during the second stage of cyclic loading (20-191 N), whereas Achillon and PARS groups stitched by No. 2 sutures all failed during the third stage of cyclic loading (20-369 N). Following 1,000 cycles, the mean elongation of CAMIR and CAMIR ${ }^{+}$restoration groups exhibited no significant differences with the Krackow restoration group, but were lower than those of the Achillon and PARS repair groups. This indicated that stitching with the CAMIR with No. 2 suture was as strong as the Krackow technique, but was still weaker than the Achillon and PARS techniques. Otherwise, augmentation 


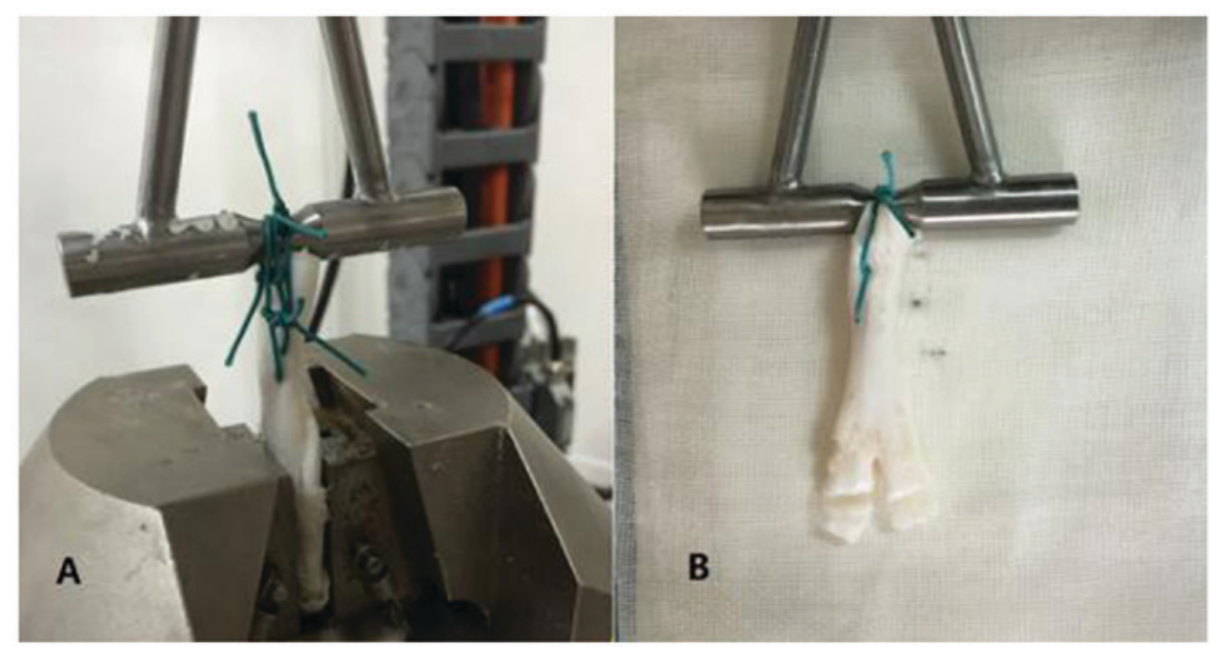

Figure 5. The number of stitch knots. (A) Three large knots, as present in Achillon and PARS techniques. (B) The channel-assisted minimally invasive repair and Krackow techniques use only 1 knot.

with lower resisted intensity sutures did not increase the strength and elongation resistance. All restorations provided reliable strength for postoperative isokinetic training.

The $90 \%$ failure rate of sutures in the current experiment was due to a rupture at the suture junction, indicating that stress was predominantly located at the suture junction. The current study revealed that Achillon and PARS are stronger compared with CAMR and Krackow as they hold one end of the Achilles tendon by three lines. The three lines divided the stress and reduced the stress concentration at the knot. The study by McKeon et al (19) also demonstrated that increasing the number of sutures increased suture strength. However, the more sutures and knots in the tendon, the greater the risk of postoperative complications (35-39). Therefore, it was deemed that best practice included selecting the surgical procedure that required fewer sutures and provided reliable strength for early passive motion of the ankle joint. CAMIR, which used only one suture, did not fail during the 20-100 N cycle, indicating that the use of CAMIR can provide reliable strength for early passive ankle joint activity.

The CAMIR suture method, which used No. 5 Ethibond sutures, failed in $86 \%$ of the samples as the suture was broken at the knot, rather than the suture that pulled the Achilles tendon. In order to test the reliability of the CAMIR suture method and increase the strength of the suture, No. 5 Ethibond sutures were selected, and it was revealed that the strength of the suturing in the fifth thread of the CAMIR-5 group was indistinguishable from the Achillon and PARS methods, which sutured with the second thread. The results suggested that the CAMIR suture strength can be increased by increasing the thickness and tensile strength of the suture, therefore this method is very reliable. The difference between the CAMIR, PARS and Achillon methods is that CAMIR has 1 line at the proximal and 1 line at the distal, with a total of 2 knots, and PARS and Achillon have 3 lines at the proximal and 3 lines at the distal, with a total of 6 knots located among the stumps. More knots in the soft tissue under the skin may lead to incision complications. In the present study, pull-out from the tendon accounted for $43 \%$ of failure of Achillon restoration, which was more prevalent than in other groups.
In this experiment, $43 \%$ of Achillon's failure was due to suture breakage, which was more prevalent than in other groups. PARS, CAMIR, and Krackow techniques all have locking sutures, which allow the structure of the locking mechanism to grip the Achilles tendon tighter when the pulling force applied to the suture increases (28). The stitching structure of Achillon uses 3 parallel lines, and there is no locking structure, which cannot convert the pulling force into grasping force (28). As the pulling force increases, the sutures cut the Achilles tendon and cause the suture to fail (28).

In the present study, Achillon and PARS restoration techniques were comprised of three sutures, whereas Krackow and CAMIR require only one suture. Although three sutures enhanced the strength of stitching, there were three large knots on the sides of the end of tendons. The present study only simulated one side of the Achilles tendon rupture as only three knots were used. In clinical practice, six knots are used as there are two broken ends when the Achilles tendon breaks. Achilles tendon rupture typically occurred $2-6 \mathrm{~cm}$ proximal to the tendon for inserting (36), where coverage of tissue and skin is not thick. Too many sutures and knots with a larger caliber of suture may increase the risk of suture reactivity, which may cause postoperative complications, such as abscess, granuloma or fistula at the incision site, and infection (37-39).

There were a number of limitations in the present investigation. Firstly, the use of porcine Achilles tendon specimens may not perfectly represent the dimensions and architecture observed in human tendons. However, porcine tendon has been used in a number of studies evaluating different fixation techniques for tendon restoration $(19,20)$. Secondly, percutaneous restorations in the present study were made using an open approach simulating the restoration technique without specialized stitching devices, which may have led to inaccurate repairing. In clinical practice, percutaneous sutures often do not expose the threading site and cannot be viewed directly. In particular, it is difficult for the three sutures in the Achillon method to pass through the maximum diameter of the Achilles tendon as in the current study, thereby weakening the suture strength. Finally, one side of the lacerated tendon ends were sutured, which has also been the case in multiple studies evaluating different fixation techniques 
for tendon restoration $(23,24)$. However, the limited number of samples is the main disadvantage of the present study. In future studies, a large number of samples are required.

In conclusion, the suture structure of CAMIR can achieve reliable suture strength with fewer stitches and knots, reaching the strength of the open Krackow restoration technique, but weaker than Achillon and PARS techniques. To a certain extent, the greater the tensile strength of the suture used, the stronger the tensile strength of the CAMIR suture structure. The intermittent reinforcement of the broken ends does not improve the suturing strength.

\section{Acknowledgements}

Not applicable.

\section{Funding}

The present study was supported by Beijing Municipal Science \& Technology Commission (grant no. Z161100000516192).

\section{Availability of data and materials}

All data generated or analyzed during the current study are included in this published article.

\section{Authors' contributions}

$\mathrm{HQ}$, YC and XJ performed the surgeries. HQ wrote the manuscript. HQ, HC, PT and LW proposed experimental theories and designed the experiment. LW trained $\mathrm{HQ}, \mathrm{YC}$, and $\mathrm{XJ}$ to use the experimental instruments. All authors read and approved the final manuscript.

\section{Ethics approval and consent to participate}

The Ethics Committee for Experiments on Animals of General Hospital of People's Liberation Army (Beijing, China) approved all procedures in the present study.

\section{Patient consent for publication}

Not applicable.

\section{Competing interests}

The authors declare that they have no competing interests.

\section{References}

1. Jozsa L, Kvist M, Balint BJ, Reffy A, Järvinen M, Lehto M and Barzo M: The role of recreational sport activity in Achilles tendon rupture. A clinical, pathoanatomical, and sociological study of 292 cases. Am J Sports Med 17: 338-343, 1989.

2. Ganestam A, Kallemose T, Troelsen A and Barfod KW: Increasing incidence of acute Achilles tendon rupture and a noticeable decline in surgical treatment from 1994 to 2013. A nationwide registry study of 33,160 patients. Knee Surg Sports Traumatol Arthrosc 24: 3730-3737, 2016.

3. Khan RJ, Fick D, Keogh A, Crawford J, Brammar T and Parker M: Treatment of acute achilles tendon ruptures. A meta-analysis of randomized, controlled trials. J Bone Joint Surg Am 87: 2202-2210, 2005.
4. Wilkins $\mathrm{R}$ and Bisson LJ: Operative versus nonoperative management of acute Achilles tendon ruptures: A quantitative systematic review of randomized controlled trials. Am J Sports Med 40: 2154-2160, 2012.

5. Deng S, Sun Z, Zhang C, Chen G and Li J: Surgical treatment versus conservative management for acute Achilles tendon rupture: A systematic review and meta-analysis of randomized controlled trials. J Foot Ankle Surg 56: 1236-1243, 2017.

6. Soroceanu A, Sidhwa F, Aarabi S, Kaufman A and Glazebrook M: Surgical versus nonsurgical treatment of acute Achilles tendon rupture: A meta-analysis of randomized trials. J Bone Joint Surg Am 94: 2136-2143, 2012.

7. Hattrup SJ and Johnson KA: A review of ruptures of the Achilles tendon. Foot Ankle 6: 34-38, 1985

8. Del Buono A, Volpin A and Maffulli N: Minimally invasive versus open surgery for acute Achilles tendon rupture: A systematic review. Br Med Bull 109: 45-54, 2014.

9. Li Q, Wang C, Huo Y, Jia Z and Wang X: Minimally invasive versus open surgery for acute Achilles tendon rupture: A systematic review of overlapping meta-analyses. J Orthop Surg Res 11: 65, 2016.

10. Lee SJ, Sileo MJ, Kremenic IJ, Orishimo K, Ben-Avi S, Nicholas SJ and McHugh M: Cyclic loading of 3 Achilles tendon repairs simulating early postoperative forces. Am J Sports Med 37: 786-790, 2009.

11. Aibinder WR, Patel A, Arnouk J, El-Gendi H, Korshunov Y, Mitgang J and Uribe J: The rate of sural nerve violation using the Achillon device: A cadaveric study. Foot Ankle Int 34: 870-875, 2013.

12. Carmont MR and Maffulli N: Modified percutaneous repair of ruptured Achilles tendon. Knee Surg Sports Traumatol Arthrosc 16: 199-203, 2008.

13. Maffulli N, Longo UG, Maffulli GD, Khanna A and Denaro V: Achilles tendon ruptures in elite athletes. Foot Ankle Int 32: 9-15, 2011.

14. Maffulli N, Longo UG, Ronga M, Khanna A and Denaro V: Favorable outcome of percutaneous repair of Achilles tendon ruptures in the elderly. Clin Orthop Relat Res 468: 1039-1046, 2010.

15. Chen H, Ji X, Tang P, Liang X and Tang P: Channel-assisted minimally invasive repair of acute Achilles tendon rupture. J Orthop Surg Res 10: 167, 2015.

16. Krackow KA, Thomas SC and Jones LC: A new stitch for ligament-tendon fixation. Brief note. J Bone Joint Surg Am 68: 764-766, 1986.

17. Calder JD and Saxby TS: Early, active rehabilitation following mini-open repair of Achilles tendon rupture: A prospective study. Br J Sports Med 39: 857-859, 2005.

18. Assal M, Jung M, Stern R, Rippstein P, Rippstein P, Delmi M and Hoffmeyer P: Limited open repair of Achilles tendon ruptures: A technique with a new instrument and findings of a prospective multicenter study. J Bone Joint Surg Am 84-A: 161-170, 2002.

19. McKeon BP, Heming JF, Fulkerson J and Langeland R: The Krackow stitch: A biomechanical evaluation of changing the number of loops versus the number of sutures. Arthroscopy 22 : 33-37, 2006.

20. Ostrander RV, Saper MG and Juelson TJ: A biomechanical comparison of modified krackow and locking loop suture patterns for soft-tissue graft fixation. Arthroscopy 32: 1384-1388, 2016.

21. Hapa O, Erduran M, Havitçioğlu H, Çeçen B, Akşahin E, Güler S and Atalay K: Strength of different Krackow stitch configurations using high-strength Suture. J Foot Ankle Surg 52: 448-450, 2013.

22. Orishimo KF, Burstein G, Mullaney MJ, Kremenic IJ, Nesse M, McHugh MP and Lee SJ: Effect of knee flexion angle on Achilles tendon force during passive dorsiflexion. J Foot Ankle Surg 47: 34-39, 2008.

23. Akizuki KH, Gartman EJ, Nisonson B, Ben-Avi S and McHugh MP: The relative stress on the Achilles tendon during ambulation in an ankle immobilizer: Implications for rehabilitation after Achilles tendon repair. Br J Sports Med 35: 329-334, 2001.

24. McMahon SE, Smith TO and Hing CB: A meta-analysis of randomised controlled trials comparing conventional to minimally invasive approaches for repair of an Achilles tendon rupture. Foot Ankle Surg 17: 211-217, 2011.

25. Cretnik A, Kosanovic M and Smrkolj V: Percutaneous versus open repair of the ruptured Achilles tendon: A comparative study. Am J Sports Med 33: 1369-1379, 2005.

26. Ebinesan AD, Sarai BS, Walley GD and Maffulli N: Conservative, open or percutaneous repair for acute rupture of the Achilles tendon. Disabil Rehabil 30: 1721-1725, 2008. 
27. Cetti R, Christensen SE, Ejsted R, Jensen NM and Jorgensen U: Operative versus nonoperative treatment of Achilles tendon rupture. A prospective randomized study and review of the literature. Am J Sports Med 21: 791-799, 1993.

28. Mark-Christensen T, Troelsen A, Kallemose T and Barfod KW: Functional rehabilitation of patients with acute Achilles tendon rupture: A meta-analysis of current evidence. Knee Surg Sports Traumatol Arthrosc 24: 1852-1859, 2016.

29. Nilsson-Helander K, Silbernagel KG, Thomeé R, Faxén E, Olsson N, Eriksson BI and Karlsson J: Acute Achilles tendon rupture: A randomized, controlled study comparing surgical and nonsurgical treatments using validated outcome measures. Am J Sports Med 38: 2186-2193, 2010.

30. Brumann M, Baumbach SF, Mutschler W and Polzer H: Accelerated rehabilitation following Achilles tendon repair after acute rupture-Development of an evidence-based treatment protocol. Injury 45: 1782-1790, 2014.

31. Clanton TO, Haytmanek CT, Williams BT, Civitarese DM, Turnbull TL, Massey MB, Wijdicks CA and LaPrade RF: A biomechanical comparison of an open repair and 3 minimally invasive percutaneous Achilles tendon repair techniques during a simulated, progressive rehabilitation protocol. Am J Sports Med 43: 1957-1964, 2015.

32. Demetracopoulos CA, Gilbert SL, Young E, Baxter JR and Deland JT: Limited-open Achilles tendon repair using locking sutures versus nonlocking sutures: An in vitro model. Foot Ankle Int 35: 612-618, 2014.
33. Heitman DE, Ng K, Crivello KM and Gallina J: Biomechanical comparison of the Achillon tendon repair system and the Krackow locking loop technique. Foot Ankle Int 32: 879-887, 2011.

34. Benthien RA, Aronow MS, Doran-Diaz V, Sullivan RJ, Naujoks R and Adams DJ: Cyclic loading of Achilles tendon repairs: A comparison of polyester and polyblend suture. Foot Ankle Int 27: 512-518, 2006.

35. Nichol SA and Silk WK: Empirical evidence of a convection-diffusion model for $\mathrm{pH}$ patterns in the rhizospheres of root tips. Plant Cell Environment 24: 967-974, 2001.

36. Cevik M: Acquired Umbilico-inguinal fistula with persistent discharge due to suture reaction: A case report and review of the literature. Case Rep Med 2012: 216345, 2012.

37. Aktas S and Kocaoglu B: Open versus minimal invasive repair with Achillon device. Foot Ankle Int 30: 391-397, 2009

38. Casha JN and Hadden WA: Suture reaction following skin closure with subcuticular polydioxanone in total knee arthroplasty. J Arthroplasty 11: 859-861, 1996.

39. Kara A, Celik H, Seker A, Uysal MA, Uzun M and Malkoc M: Granuloma formation secondary to Achilles tendon repair with nonabsorbable suture. Int J Surg Case Rep 5: 720-722, 2014. 